\title{
Efficacy and Limitation of F-18-fluorodeoxyglucose Positron Emission Tomography during Fasting to Assess Myocardial Viability in the Acute Phase of Myocardial Infarction
}

\author{
Hiroshi Nonogi, Shunichi Mryazaki, Yoichi Goto, Yoshio IshidA*, \\ Toshiisa UeHARA** and Tsunehiko NishIMURA**
}

\begin{abstract}
Objective: The present study was designed to determine the ability of positron emission tomography (PET) to assess myocardial viability and ischemia in acute myocardial infarction (MI) after reperfusion therapy (thrombolysis and/or coronary angioplasty). Methods: PET with fluorine-18-labeled fluorodeoxyglucose (FDG) under fasting conditions and thallium-201 singlephoton emission computed tomography (TL) were analyzed in 21 patients one week following MI. Myocardial viability was also assessed by regional wall motion using serial analysis of 2-D echocardiography or left ventriculography. Results: Marked uptake of FDG together with a residual perfusion defect were observed in the infarct region in all patients one week post $\mathrm{MI}$. However, in 9 of the 21 patients, the infarct-related coronary artery had no significant stenosis after reperfusion therapy and remained patent in one month post MI suggesting no myocardial ischemia. In contrast, in 4 of the 21 patients the regional wall motion was akinetic and there was a complete defect observed with TL imaging at one month post MI, indicating no viability in the infarct region. Conclusions: PET using fasting FDG at one week post MI had a limitation to predict myocardial viability or ischemia.
\end{abstract}

(Internal Medicine 37: 653-661, 1998)

Key words: ischemia, scintigram, glucose, reperfusion

\section{Introduction}

The accurate quantitative assessment of viable myocardium is important to determine subsequent therapeutic strategies in patients with acute myocardial infarction (MI) who underwent coronary reperfusion therapy. However, due to the presence of stunned or hibernating myocardium after the restoration of blood flow, it is difficult to differentiate injured but viable from necrotic tissue on the basis of regional contractile function. Recently, positron emission tomography (PET) with a glucose analogue, fluorine-18-fluorodeoxyglucose (FDG), was shown to detect viable myocardium metabolically in regions of severe hypoperfusion and dysfunction in patients with coronary artery disease $(1,2)$. Previous experimental and clinical studies have demonstrated that PET can detect residual viable tissue despite reduced blood flow and impaired contractile function $(3,4)$. In the fasted state, ischemic tissue showed an increased accumu- lation of FDG, while normal or necrotic tissue showed less uptake. However, in patients with MI treated with thrombolysis or coronary angioplasty, the usefulness of fasting FDG-PET in the assessment of myocardial viability remains unclear. Therefore, the purpose of this study was to assess the ability of FDGPET imaging during fasting to detect myocardial viability or ischemia one week after reperfusion therapy in patients with MI. Regional contractile function as determined using twodimensional echocardiography one month post MI was used as gold standard for the viability of the infarct-related region whereas regional myocardial perfusion was assessed using Thallium-201 (TL) imaging.

\section{Methods}

\section{Patients}

Twenty-one patients who were admitted to the coronary care

From the Division of Cardiology, the Department of Internal Medicine, National Cardiovascular Center, Suita, *the Department of Radiology, National Cardiovascular Center, Osaka and **the Department of Tracer Data Analysis, Biomedical Research Center, Osaka University, Osaka

Received for publication November 10, 1997; Accepted for publication May 19, 1998

Reprint requests should be addressed to Dr. Hiroshi Nonogi, the Division of Cardiology, the Department of Internal Medicine, National Cardiovascular Center, 5-7-1 Fujishirodai, Suita, Osaka 565-8565 
unit of our institute within 24 hours of the onset of acute myocardial infarction were enrolled in this study. Patients with diabetes mellitus, hemodynamic instability or complex arrhythmia were excluded from the present study. Diagnosis of acute myocardial infarction was based on the presence of at least two of the following standard three criteria; 1) typical chest pain persisting for at least 30 minutes; 2) ST-segment elevation lasting more than 30 minutes in at least 2 leads of the standard 12-lead electrocardiogram (ECG); and 3) serial elevation of serum creatine kinase. There were 16 males and 5 females with a mean age (SD) of $63+/-9$ years (range 42 to 78 ). Thirteen patients presented with anterior and 8 with inferior myocardial infarction. Sixteen patients developed Q waves in the infarct-related ECG leads (Table 1). All patients underwent urgent coronary angiography and 15 patients underwent intracoronary thrombolysis using tissue-type plasminogen activator (Hapase KOWA inj, KOWA Co., Nagoya) which was administered through the coronary catheter at a rate of 160,000
$\mathrm{U} / \mathrm{min}$ to a maximum of $6,400,000 \mathrm{U}$. Two patients underwent primary coronary angioplasty, and 4 patients did not undergo reperfusion therapy (spontaneous recanalization in 3 patients and delayed admission 12 hours after the onset). Seven of the 8 patients with unsuccessful thrombolysis underwent rescue percutaneous transluminal coronary angioplasty (PTCA). Mean left ventricular ejection fraction determined by contrast left ventriculography was averaged $48+1-12 \%$ in all 21 patients after the procedure.

Each subject gave written informed consent for the PET study approved by the National Cardiovascular Center Clinical Study Committee.

\section{Coronary angiography and left ventriculography}

Selective coronary angiography and contrast left ventriculography were performed immediately after admission and 1 month after the onset of MI. Significant coronary stenosis was defined as a reduction in the luminal diameter of $\geq 70 \%$. The

Table 1. Clinical Characteristics

\begin{tabular}{|c|c|c|c|c|c|c|c|c|c|c|c|}
\hline Group & & Age & Sex & MI & VD & $\begin{array}{l}\text { Culprit } \\
\text { vessel }\end{array}$ & Qwave & $\begin{array}{l}\% \mathrm{DS} \\
\text { after }\end{array}$ & $\begin{array}{r}\% \mathrm{DS} \\
1 \mathrm{M}\end{array}$ & $\begin{array}{c}\text { Collateral } \\
\text { before }\end{array}$ & $\begin{array}{c}\text { Collateral } \\
1 \mathrm{M}\end{array}$ \\
\hline \multirow[t]{7}{*}{ Group 1} & 1 & 62 & $\mathrm{M}$ & INF & 1 & $\mathrm{RCA}$ & $Q$ & 62 & 48 & 2 & 0 \\
\hline & 2 & 57 & $\mathbf{M}$ & ANT & 3 & LAD graft & $\mathrm{Q}$ & 50 & 75 & 2 & 0 \\
\hline & 3 & 60 & $\mathrm{M}$ & INF & 2 & $\mathrm{RCA}$ & $\mathrm{N}$ & 0 & 25 & 1 & 0 \\
\hline & 4 & 78 & $\mathrm{M}$ & ANT & 1 & LAD & Q & 37 & 40 & 2 & 0 \\
\hline & 7 & 72 & $\mathrm{~F}$ & ANT & 1 & LAD & $\mathrm{Q}$ & 39 & 25 & 0 & 0 \\
\hline & 8 & 60 & $\mathrm{M}$ & ANT & 1 & LAD & $\mathrm{N}$ & 30 & 25 & 2 & 0 \\
\hline & 9 & 63 & $\mathrm{M}$ & ANT & 1 & LAD & $\mathrm{Q}$ & 25 & 58 & 1 & 0 \\
\hline
\end{tabular}

\begin{tabular}{|c|c|c|c|c|c|c|c|c|c|c|c|}
\hline \multicolumn{2}{|c|}{ Mean +/-SD } & \multicolumn{2}{|c|}{657} & \multicolumn{3}{|c|}{$\begin{array}{ll}1.3 & 0.7\end{array}$} & \multicolumn{2}{|r|}{3224} & \multicolumn{2}{|l|}{4018} & \multirow[b]{2}{*}{0} \\
\hline \multirow[t]{7}{*}{ Group 2} & 10 & 59 & M & INF & 1 & RCA & $Q$ & 75 & 90 & 2 & \\
\hline & 11 & 50 & M & ANT & 1 & LAD & Q & 90 & 99 & 1 & 0 \\
\hline & 12 & 42 & M & ANT & 1 & LAD & $\mathrm{Q}$ & 75 & 75 & 2 & 0 \\
\hline & 13 & 57 & M & ANT & 2 & LAD & $\mathrm{N}$ & 90 & 90 & 0 & 0 \\
\hline & 14 & 52 & $\mathrm{~F}$ & INF & 1 & $\mathrm{RCA}$ & Q & 90 & 90 & 0 & 0 \\
\hline & 17 & 52 & M & INF & 2 & RCA & $\mathrm{Q}$ & 75 & 50 & 2 & 0 \\
\hline & 18 & 67 & M & ANT & 2 & LAD & $\mathrm{Q}$ & 90 & 50 & 0 & 0 \\
\hline
\end{tabular}

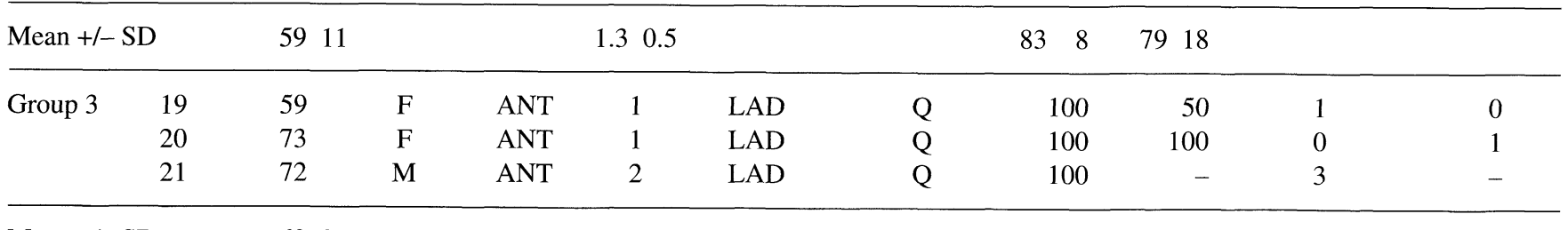

$\begin{array}{llllllll}\text { Mean +l-SD } & 68 & 8 & 1.3 & 0.6 & \text { Q:16N:5 } & 100 & 0\end{array}$

MI: myocardial infarction, VD: extent of coronary vessel disease, \%DS: diameter stenosis after reperfusion therapy (after) and in the convalescent phase (1M), Collateral: collateral to the infarct area before reperfusion therapy (before) and in the convalescent phase (1M), $\mathrm{M}$ : male, F: female, INF: Inferior, ANT: anterior, RCA: right coronary artery, LAD: left anterior descending artery, Q: Q wave infarction, N: non$\mathrm{Q}$ wave infarction, 1M: 1 month after the onset of acute myocardial infarction, -: not obtained. 
infarct-related artery was identified from the ECG and the location of wall motion abnormalities by ventriculography or echocardiography. Success of the thrombolytic therapy in the infarct-related artery was defined angiographically as achieving thrombolysis in the myocardial infarction trial (TIMI) flow grade 3 (5). Coronary angioplasty success was defined angiographically as achieving less than 50\% diameter residual stenosis. The percent diameter stenosis was calculated by the caliper method. Collaterals to the infarct area were graded according to Cohen and Rentrop as follows: grade $0=$ no collaterals; 1 = poor; $2=$ fair; $3=\operatorname{good}(6)$. Left ventricular ejection fraction was calculated from the ventriculogram using the area-length method.

Patients were classified into 3 groups according to their coronary reperfusion state immediately after reperfusion therapy (Table 1); 9 patients with no significant residual stenosis after successful reperfusion were placed into Group 1 (diameter stenosis $99.8+/-0.4 \%$ before reperfusion to $31.9+/-23.7 \%$ after reperfusion, $\mathrm{p}<0.01$ and $39.6+/-24.7 \%$ at one month post MI), 9 patients having a residual significant stenosis in the acute phase were placed into Group 2 (diameter stenosis 93.1+/$10.8 \%$ before reperfusion to $83.3+1-7.9 \%$ after reperfusion, $\mathrm{p}<0.01$ and $78.8+/-18.0 \%$ stenosis at one month post MI), and 3 patients with remaining total occlusion in the acute phase into Group 3.

\section{Positron emission tomography}

The PET study in acute phase of MI was performed on average $8+/-3$ days after the onset. Because one patient (case 13) refused the PET study in the convalescent phase, the PETstudy was repeated in 20 patients on average $41+/-13$ days after the onset of MI.

PET was performed with a whole-body, multislice positron camera (Headtome 4, Shimazu, Kyoto). It provided 15 slices at 7-mm intervals with an effective resolution of approximately 5 $\mathrm{mm}$. Each patient was fasted at least 5 hours to maintain a metabolic steady state during the study. A transmission scan was performed for 15 minutes for accurate correction of photon attenuation. Then, $5 \mathrm{mCi}$ of FDG was injected intravenously at rest. Approximately 40 minutes later, glucose metabolic imaging was performed for 12 minutes. Immediately after the first scan, the patient was moved $7 \mathrm{~mm}$ in the axial direction and the second scan was performed. These two scans provided a total of 15 contiguous transaxial slices ( $7 \mathrm{~mm}$ spacing). The results of the FDG imaging were determined by two observers blinded to both the TL image and the clinical data and were scored as follows: $0=$ no FDG uptake; $1=$ less than liver uptake; $2=$ equal or more than liver uptake; $3=$ markedly more than liver uptake. Disagreement between the 2 observers was resolved by consensus.

\section{Thallium -201 chloride study}

A resulting TL scan with single photon emission tomographic images was performed before the PET study on the same day in 18 patients. Series of transaxial slices were reconstructed with a filtered back projection without using attenua- tion correction. Oblique tomograms parallel to the long and short axes of the left ventricle were also reconstructed.

One month after the onset of MI, graded bicycle exercise was performed in 19 patients starting at $25 \mathrm{~W}$ with an increment of $25 \mathrm{~W}$ every 2 minutes. At peak exercise, $2.5 \mathrm{mCi}$ of TL was injected, and the exercise was continued for another 1 minute. The stress TL scan began within 10 minutes of the tracer injection. Four hours later, a delayed TL scan was performed. An additional $1 \mathrm{mCi} \mathrm{TL}$ was reinjected immediately after the redistribution study and the images were obtained within 10 to 15 minutes of the TL injection by use of the same imaging protocol.

Two experienced observers who were unaware of the clinical and PET data, scored the TL uptake in the infarct region using a 4-point grading system $(0=$ normal, $1=$ mild but definite defect, $2=$ severe defect, $3=$ complete defect) (7). At one month post MI, a defect was considered fixed if the regional TL uptake score remained unchanged between the initial and the reinjection images.

\section{Two-dimensional echocardiography}

Serial assessment of regional systolic contractile function was performed using two-dimensional echocardiography in all patients. For analysis of regional function, the left ventricle was divided into 9 regions. Regional systolic function was quantified as a wall motion index; $1=$ normal, $2=$ hypokinetic, $3=$ akinetic, $4=$ dyskinetic and $5=$ aneurysmal (8). The wall motion analyses were performed by two observers blinded to both the PET and the clinical findings. Disagreement in the scoring was resolved by consensus. A regional wall motion score was calculated for each patient as the sum of the individual segment scores. Improvement in regional function was defined by a change of at least one grade in the wall motion score (4).

\section{Examinations in the chronic phase}

For 3 of 4 patients (cases 16, 19, 20) with no functional recovery in echocardiographic analysis, echocardiography, TL scintigraphy and FDG-PET study were performed 3 months after the onset of MI.

\section{Definition of myocardial viability}

The gold standard for assessing myocardial viability used in this study was based on echocardiographic findings indicating more than one score improvement or hypokinesis of the infarctrelated region one month after the onset of MI. On the other hand, absence of viability was defined as persistent regional akinesis or dyskinesis accompanied by a fixed TL defect at rest or after reinjection.

\section{Statistics}

Values are presented as mean $+/-S D$. Paired differences were analyzed with a paired $t$ test. Values of $p<0.05$ were considered statistically significant. 


\section{Results}

\section{FDG-PET uptake score}

Tables 1 and 2 summarize the findings in all 21 patients. An enhanced FDG uptake in the fasting state was observed in the infarct segment in all 21 patients at $8+/-3$ days (acute phase) after the onset of MI. A representative case in Group 1 is shown in Fig. 1 and the serial changes in FDG uptake for each group is summarized in Fig. 2.

In the 9 patients of Group 1 (no significant stenosis after successful reperfusion therapy), a positive FDG uptake was observed in all cases in the acute phase and in 7 of the 9 patients $(78 \%)$ in the convalescent phase (Fig. 2). Since the infarctrelated artery showed no significant stenosis even in the convalescent phase, no ischemia should have been present in the reperfused region. Thus, these findings demonstrate a paradoxical regional enhancement of FDG uptake under fasting conditions without ischemia after reperfusion therapy.
In the 9 patients of Group 2 having significant residual stenosis after successful reperfusion in the acute phase, enhanced FDG uptake was observed in both the acute and the convalescent phase. In one (case 18) of the 9 patients, FDG uptake was not observed in the convalescent phase suggesting viability without ischemia in the infarct region.

In the 3 patients of Group 3 with total occlusion in the acute phase, FDG uptake was observed in all 3 patients in the acute phase, whereas it was decreased but still observed in the convalescent phase.

Thus, a positive FDG uptake under fasting conditions was observed in all patients at 1 week post MI regardless of stenosis of the infarct-related artery. There was a tendency for less FDG uptake at 1 month post MI in all group, but FDG uptake was still present in all but 3 patients.

\section{TL defect score}

In 18 of the 21 patients, resting TL imaging was performed to assess the myocardial perfusion in the segments with en-

Table 2. Echocardiographic, TL Scintigraphic and PET Findings

\begin{tabular}{|c|c|c|c|c|c|c|c|c|c|c|c|}
\hline \multirow{6}{*}{ Group 1} & 2 & $\mathrm{~F}$ & $S$ & 15 & 14 & $\mathrm{a}$ & hypo & - & 1 & 3 & 2 \\
\hline & 3 & $\mathrm{~F}$ & $S$ & 18 & 16 & $\mathrm{a}$ & hypo & 2 & 1 & 3 & 0 \\
\hline & 4 & $\mathrm{~F}$ & $S$ & 16 & 11 & $\mathrm{a}$ & hypo & 2 & 1 & 3 & 2 \\
\hline & 7 & $\mathrm{~F}$ & $S$ & 19 & 17 & a & hypo & - & 2 & 2 & 2 \\
\hline & 8 & - & $S$ & 14 & 11 & $\mathrm{a}$ & hypo & 1 & 1 & 2 & 1 \\
\hline & 9 & $\mathrm{~F}$ & $S$ & 15 & 15 & $\mathrm{a}$ & $\mathrm{a}$ & 3 & 3 & 1 & 0 \\
\hline
\end{tabular}

\begin{tabular}{|c|c|c|c|c|c|c|c|c|c|c|c|c|}
\hline \multirow[t]{6}{*}{ Group 2} & 10 & $\mathrm{~S}$ & - & 13 & & 11 & $\mathrm{a}$ & hypo & 2 & 1 & 2 & 1 \\
\hline & 12 & - & - & 13 & & 11 & $\mathrm{a}$ & hypo & 3 & 1 & 2 & 1 \\
\hline & 13 & - & - & 19 & & 16 & a & hypo & 2 & 1 & 2 & - \\
\hline & 14 & $\mathrm{~S}$ & - & 17 & & 13 & $\mathrm{a}$ & hypo & 3 & 2 & 2 & 2 \\
\hline & 17 & - & - & 17 & & 17 & $\mathrm{a}$ & hypo & 3 & 2 & 2 & 2 \\
\hline & 18 & $S$ & - & 11 & & 10 & hypo & hypo & 3 & - & 3 & 0 \\
\hline
\end{tabular}

\begin{tabular}{llllllllllllllll}
\hline Mean + l-SD & 15 & 3 & 14 & $2 *$ & 2.6 & 0.5 & 1.6 & $0.7 *$ & 2.0 & 0.5 & 1.3 & 1.0
\end{tabular}

\begin{tabular}{|c|c|c|c|c|c|c|c|c|c|c|c|}
\hline \multirow[t]{2}{*}{ Group 3} & 19 & - & $\mathrm{F}$ & 17 & 22 & $\mathrm{a}$ & $\mathrm{a}$ & 3 & 3 & 3 & 1 \\
\hline & 21 & - & - & 12 & 11 & $\mathrm{a}$ & hypo & 2 & 1 & 2 & 2 \\
\hline Mean $+/$ & & S:7 F:8 & S:8 F:1 & 153 & 166 & & & 2.70 .6 & $2.3 \quad 1.2$ & 2.30 .6 & $\begin{array}{ll}1.5 & 0.7\end{array}$ \\
\hline
\end{tabular}

ICT: intracoronary thrombolysis, PTCA: coronary angioplasty, S: success, F: failure, Echo: echocardiographic wall motion score, AC: acute phase, 1M: 1 month after the onset of acute myocardial infarction, Reg: regional asynergy in the infarct-related region, TL: 201-thallium uptake score at rest or reinjection, PET: fasting FDG uptake score, hypo: hypokinesis, a: akinesis, -: not obtained. *p<0.05, \#: p<0.01 vs AC. 
FDG

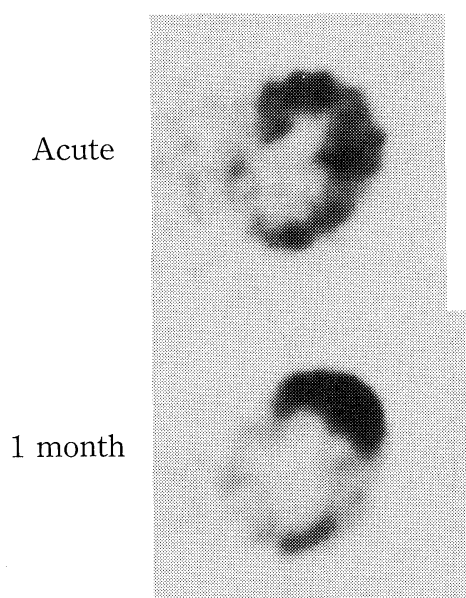

TL-EX

TL-RD

Rest (Re-inj)

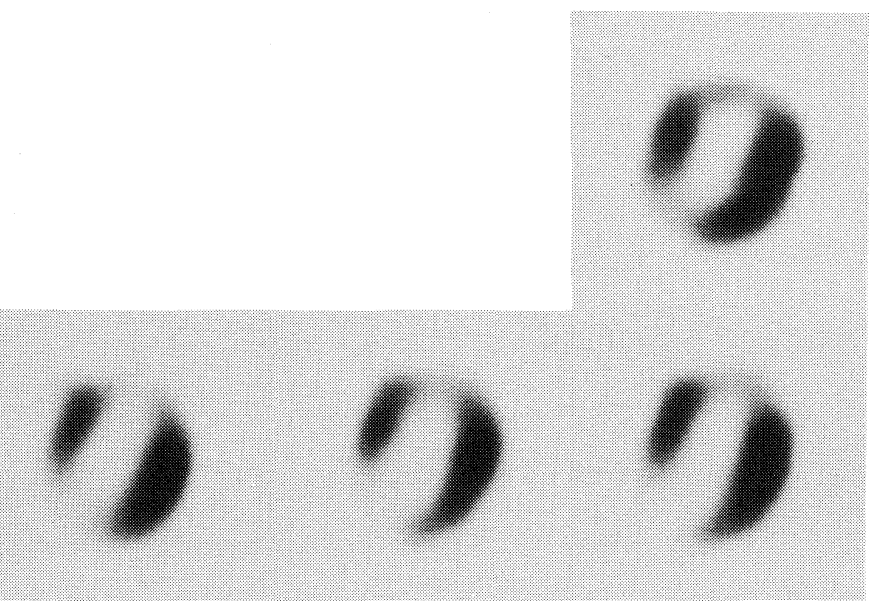

Figure 1. A representative case (case 4); Cross-sectional images after administration of F-18 deoxyglucose (FDG) and Thallium-201 (TL) 1 week (Acute, in upper panel) and 1 month (lower panel) after the onset of symptoms. He underwent intracoronary thrombolysis and rescue coronary angioplasty 5 hours after the onset with successful reperfusion extending to the convalescent phase. There is decreased TL uptake in the anterior segment of the left ventricle and the FDG image reveals enhanced glucose utilization in the same segment in the acute phase. These findings show a paradoxical enhancement of FDG. After 1 month similar images were obtained (lower panel), whereas no significant coronary stenosis was present and there was an improvement of asynergy in the infarct region was observed. TL-EX: TL image immediately after exercise, TL-RD delayed TL image 4 hours after exercise, Rest (Re-inje): resting TL image 1 week post MI or TL reinjection image 1 month post MI.
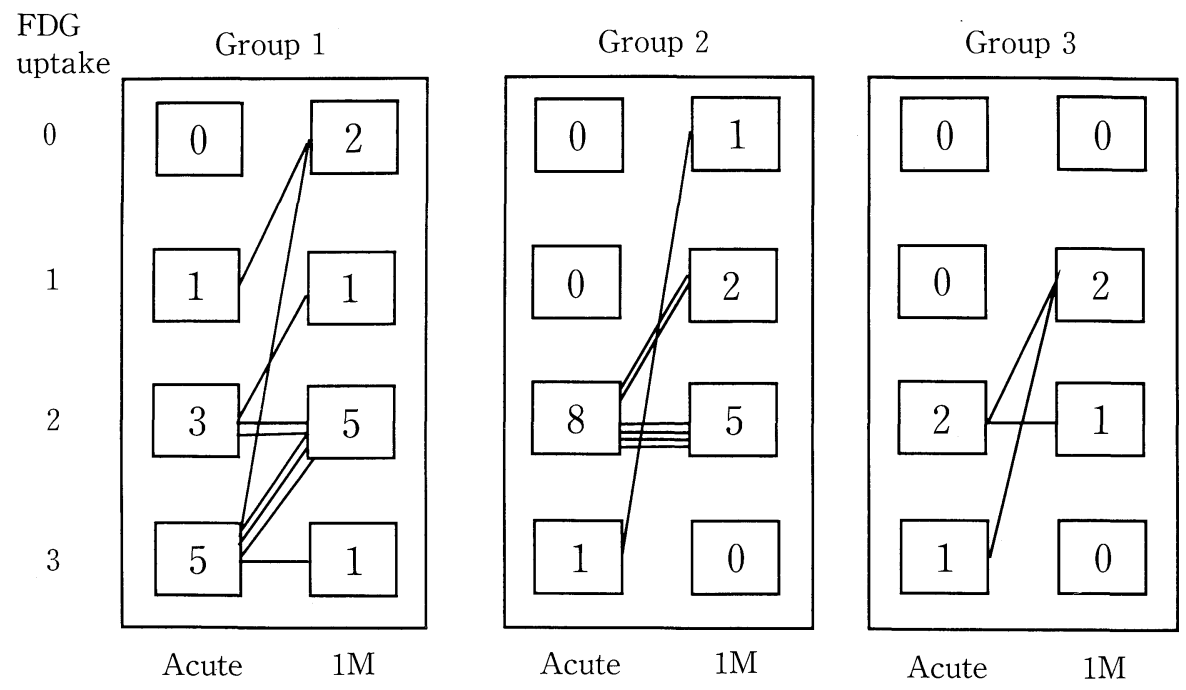

Figure 2. FDG uptake scores in the acute ( 1 week) and the convalescent phase (1 month) are compared among 3 groups classified according to patency state in the infarctrelated coronary artery in the acute phase. Positive FDG uptake was observed in all patients at 1 week post MI regardless of coronary perfusion status. Also the serial changes in FDG uptake were not specifically influenced by the coronary perfusion status. 

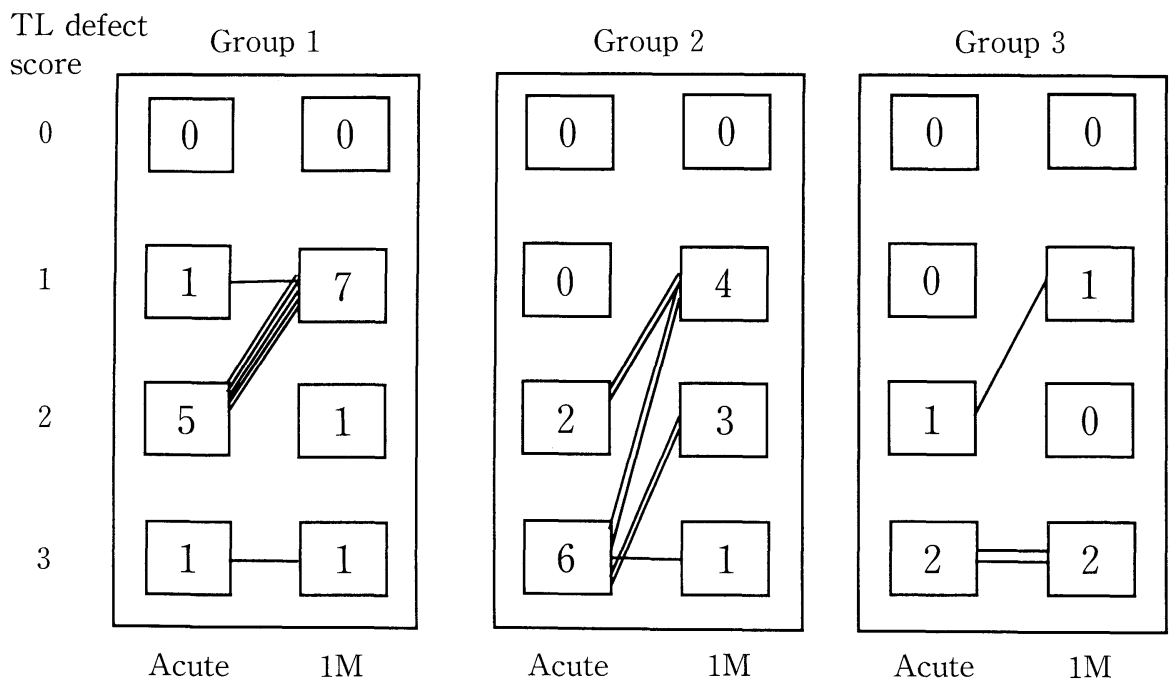

Figure 3. The prevalence of TL defect scores between the acute (1 week) and the convalescent phase (1 month) is compared among 3 groups classified according to patency state in the infarct-related coronary artery in the acute phase. TL defect score improved or mild in the convalescent phase except 4 cases with complete defect (1 patient in Group 1, 1 in Group 2, 2 in Group 3).

hanced FDG uptake. Serial changes in the TL defect score are shown in Table 1 and Fig. 3.

In Group 1, the TL defect score improved in the infarct region from 1 week to one month post MI in 5 of the 9 patients. A partial TL defect was seen in 8 of the 9 patients, indicating some perfusion in the infarct region at 1 month post MI. In one (case 9 ) of the 9 patients, the TL scintigram showed a complete TL defect with no uptake of FDG-PET in the convalescent phase, indicating no viability in the infarct region.

In Group 2, the observation of FDG uptake but with TL perfusion defect at 1 week post MI suggested metabolic viability combined with myocardial ischemia in the infarct region (Figs. 2, 3). The TL defect score improved in the infarct region in 7 of the 8 patients in the convalescent phase, indicating improved myocardial perfusion. In one (case 16), a fixed and complete TL defect with a positive FDG uptake was observed at 1 month post MI.

In Group 3, complete TL defects remained unchanged in the convalescent phase in 2 of the 3 patients. In one (case 21), the TL defect score improved from severe to mild in the convalescent phase, indicating myocardial viability.

\section{Regional wall motion and viability}

In Group 1, the regional echocardiographic score improved in the convalescent phase ( $p<0.01$, Fig. 4). Eight of the 9 patients showed an improvement in regional function as defined by a change of at least one grade in wall motion score (4), suggesting residual myocardial viability in these patients. In contrast, one patient (case 9) had no improvement in regional wall motion, a large number of $Q$ waves and a complete TL defect indicating no viability in the infarct region (Fig. 5, closed circle). However, a positive FDG uptake was observed, sug-
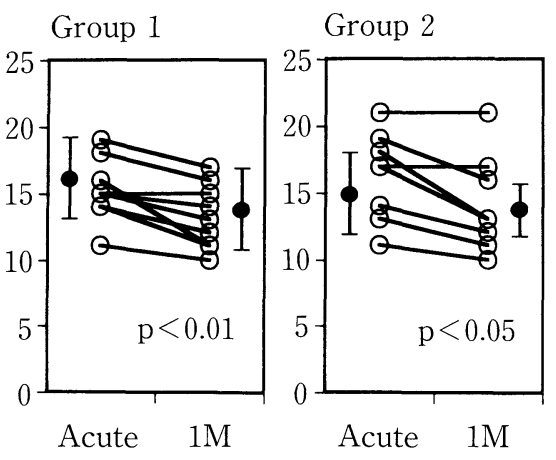

Group 3

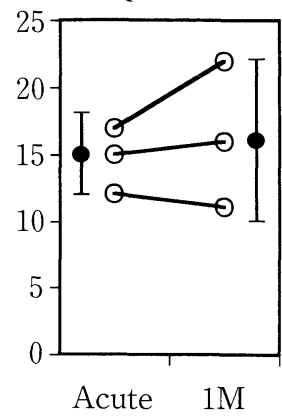

Figure 4. Changes in echocardiographic regional wall motion scores between acute ( 1 week) and the convalescent phase ( 1 month).

gesting an overestimation of viability by this technique in this patient.

In Group 2, an improvement of more than 1 echocardiographic score or hypokinesis in the infarct region in the convalescent phase indicated residual myocardial viability in 8 of the 9 patients. In the remaining patient (case 16), no improvement of regional wall motion and a large number of $\mathrm{Q}$ waves with a fixed TI defect were observed, suggesting no viability in the infarct region (Fig. 5, closed square). However, in this case, FDG uptake was still observed in the infarct region.

In Group 3, the regional echocardiography score worsened in the convalescent phase and akinesis was observed in the infarct region despite a positive FDG uptake in 2 patients (Fig. 5, closed triangle). One patient (case 21) who had a welldeveloped collateral vessel from the right coronary artery to the left anterior descending artery did not undergo reperfusion 
therapy due to the late admission 17 hours after the onset. Nonetheless, the regional echocardiography score improved in the convalescent phase in this patient (Fig. 5, open triangle).

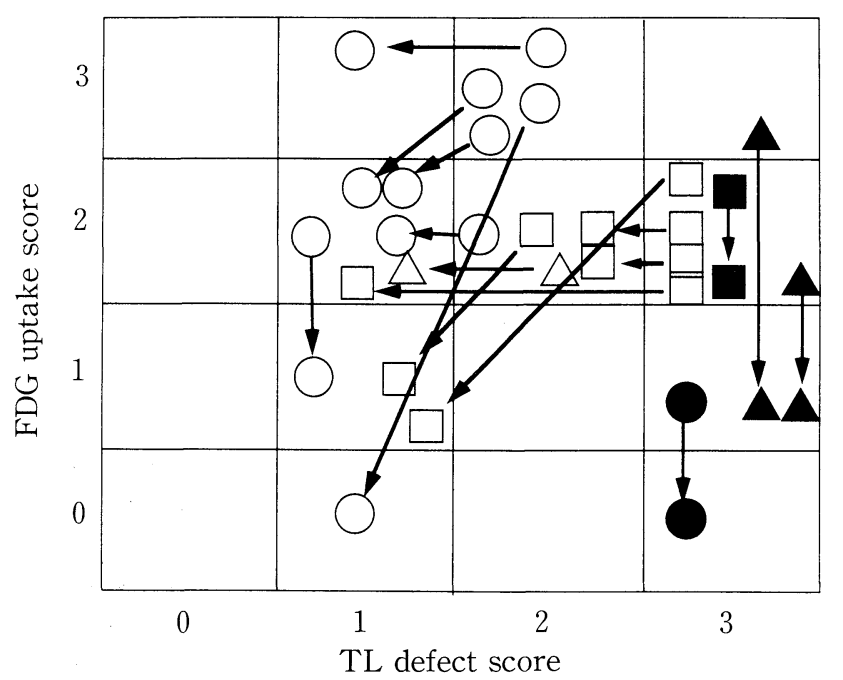

Thus, the serial changes in FDG uptake were not specifically influenced by the coronary perfusion status. The fasting FDG overestimated viability in 4 of 21 patients (19\%) in the acute phase and in 3 of $20(15 \%)$ in the convalescent phase 1 month after the onset (Fig. 5, closed symbols). Four patients without functional recovery in the convalescent phase, regional echocardiography showed still akinesis in the infarct region 3 months after the onset (chronic phase), suggesting no viability. Three of the 4 patients showed severe fixed defect in TL scintigraphy and attenuation of uptake in the fasting FDG-PET in the chronic phase (Fig. 6). The fasting FDG overestimated ischemia in 12 of 21 patients (57\%) in the acute phase and in 10 of 20 patients $(50 \%)$ in the convalescent phase.

Figure 5. Changes in both FDG uptake score and TL defect score in 16 patients: Group 1 (circle), Group 2 (square), Group 3 (triangle). All cases show positive FDG uptake in acute phase. Open symbols show clinical viability and closed symbols no clinical viability with complete defect in TL scintigram despite positive uptake in FDG in acute phase.

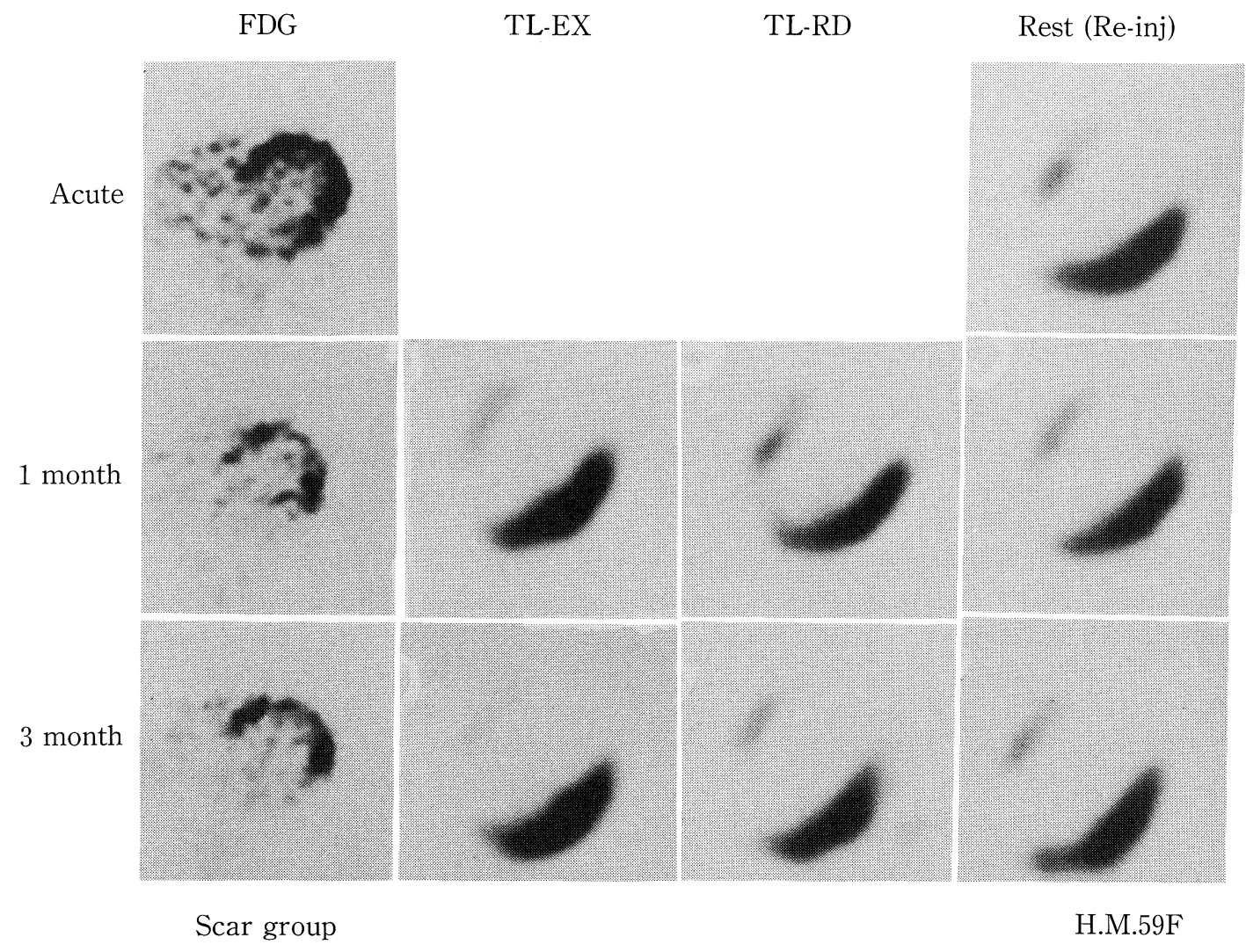

Figure 6. A representative case for Group 3 (case 19); Cross-sectional images after administration of F-18 deoxyglucose (FDG) and Thallium-201 (TL) 1 week (Acute, in upper panel), 1 month (middle panel) and 3 months (lower panel) after the onset of symptoms. She underwent primary PTCA with unsuccessful reperfusion. TL uptake of rest (acute phase) and reinjection (1 and 3 months) show severe fixed defect in the anterior segment of the left ventricle. FDG-PET image reveals enhanced glucose utilization in the same segment in the acute phase. However, FDG uptake is markedly attenuated both in the convalescent and the chronic phase. 


\section{Discussion}

The present study indicates that the fasting FDG uptake is enhanced in the infarct region in all patients in the acute phase (1 week post MI) of MI independent of stenosis severity or patency of the infarct vessel, and TL-determined myocardial perfusion. Also, this study demonstrated that use of fasting FDG-PET image to assess myocardial viability did not correlate well with viability assessed using echocardiography and overestimated myocardial ischemia in the infarct region in the convalescent phase ( $41 \pm 13$ days post MI).

\section{FDG in the acute phase of MI}

Several experimental studies described increases in glucose utilization in reperfused, dysfunctional myocardium $(3,9,10)$. These reports suggested that augmented uptake of FDG under fasting conditions identifies ischemically compromised but viable tissue. These studies were performed from 30 minutes to 3 hours after reperfusion in which the infarct region still contained reversibly injured cells. Schwaiger et al performed PET with FDG and N-13 ammonia in patients within 72 hours after the onset of acute MI (4). They suggested that PET might allow early identification of viable but jeopardized tissue. However, in their previous experimental study, an enhanced uptake of FDG was observed 24 hours after reperfusion without residual stenosis and FDG uptake normalized at 1 week after reperfusion (3). They suggested that impaired fatty acid oxidation and a shift of substrate metabolism toward glucose utilization were observed in reversibly injured myocardium to meet the energy requirement of these surviving cells. Therefore, an enhanced uptake of FDG in the fasted state within 1 week after the onset of MI may indicate recent myocardial injury, not necessarily ongoing myocardial ischemia. To avoid this problem, the PET measurements were performed 1 week after the onset of MI in the present study. At this time, myocardial irreversible injury from the initial MI should be completed (3). Nonetheless, in the present study, an enhanced uptake of FDG in the fasted state was still observed in the infarct region in all patients in this early phase independent of coronary perfusion status.

\section{Possible mechanisms in enhanced FDG uptake in the fasted state in AMI}

An infarct region may contain patchy viable tissue or a metabolically active border zone surrounding a necrotic area with hypoperfusion due to residual coronary stenosis as in Groups 2 and 3. In this situation, the enhanced FDG uptake in the fasted state may reflect ongoing ischemia one week post MI.

Alternatively, stunned myocardium in the acute phase may have enhanced glycolytic metabolism $(3,9,11)$. After exerciseinduced ischemia or after the resolution of segmental blood flow defects are resolved, FDG uptake remains augmented (11). Schwaiger et al suggested that such metabolic indices could identify viable but ischemically injured tissue 24 hours after complete reperfusion (3). They demonstrated prolonged abnormalities of fatty acid metabolism and enhanced utilization of glucose in postischemic myocardium as demonstrated by segmental abnormal clearance kinetics of 11-C-labeled palmitate (12). These findings suggest that postischemic myocardium metabolizes glucose in preference to fatty acid during the fasted state. However, others showed that fatty acids are the preferred metabolic substrate in the postischemic heart (13). Liedtke et al showed that myocardial accumulation of 14Cdeoxyglucose does not reflect glycolysis during reperfusion (14). They suggested that the myocardial kinetics of deoxyglucose are not specific for myocardial glucose metabolism. Thus, the assessment of myocardial metabolism after reperfusion remains controversial.

A further consideration is that accumulation of polymorphonuclear leukocytes in reperfused myocardium may be responsible for FDG uptake $(15,16)$. Sebree et al showed in an experimental study that the uptake of $\mathrm{C}-14$ deoxyglucose was not correlated with the extent of necrosis after reperfusion, whereas a good inverse correlation was observed between the TL defect and the percent of myocardial necrosis (17). In the present study, FDG uptake was markedly diminished in the chronic phase, whereas, TL scintigram remained severe fixed defect in 3 patients without functional recovery (Fig. 6). These findings suggested that the glucose uptake observed in the infarct region during the acute phase might be related to the metabolism of other cells such as leukocytes, in addition to viable myocytes.

\section{FDG in the convalescent phase of MI}

An uptake of FDG-PET during the fasted state was observed in 17 of the 20 patients in the convalescent phase. In 7 of these 17 patients, there was no significant stenosis in the infarctrelated artery and the infarct region showed hypokinesis, which suggests viability and no ongoing ischemia in the infarct region. In $3(18 \%)$ of the 17 patients, persistent regional akinesis and a complete TL perfusion defect were observed, indicating a scar in the infarct region (Fig. 4, closed symbols). Thus, in these 10 patients, FDG-PET appears to disagree with other estimates of ischemia. In 4 of 20 patients (closed symbols in Fig. 4), clinical findings including large numbers of Q-wave, persistent regional akinesis or dyskinesis, and a fixed TL defect indicated no myocardial viability. However, 3 of these 4 patients showed FDG uptake in the infarct region, suggesting an overestimation of myocardial viability using the FDG uptake in the fasted state. Marwick et al showed similar findings in PET with fasting FDG which overestimated the extent of tissue viability in as many as $32 \%$ of myocardial segments (18).

\section{Fasting vs. glucose loading FDG PET}

In the thrombolytic era, a residual ischemia affects short- and long-term prognosis after thrombolys due to residual stenosis or reocclusion in the infarct-related lesion (19). For such patients, a more aggressive management should be warranted to improve prognosis. Therefore, the differentiation between infarcted and ischemically-compromized but viable myocardium with significant stenosis in the infarct-related artery has become 


\section{PET in Acute Myocardial Infarction}

of major clinical importance. To detect ischemic but metabolically active regions, the fasting FDG uptake is useful. The fasting FDG-PET is preferred to accentuate the difference between ischemic tissue with increased accumulation of FDG and normal or scar tissue with less uptake. Conversely, oral glucose loading is preferred to identify viable myocardium independent of myocardial ischemia. To detect myocardial ischemia and viability in the infarct region, the fasting FDGPET was employed in the present study.

However, the present study suggests the overestimation of myocardial ischemia and viability in the fasting FDG-PET one month after MI. Recent studies performed with glucose loading and NH3 PET demonstrated a more accurate assessment of the amount of viable tissue $(16,18)$. Thus, further studies are necessary to determine which technique is best for assessing myocardial viability with or without ischemia.

\section{Study limitations}

Segmental function was studied by echocardiography because this permitted serial follow-up studies. Although contrast ventriculography may be more accurate, two-dimensional echocardiography compares well with contrast ventriculography in assessing serial changes in regional wall motion (4).

In summary, the evaluation of regional glucose metabolism by FDG-PET in a fasting state and relative distribution of blood flow by TL scintigraphy at rest early after the onset of acute MI showed the limitation to distinguish necrotic from reversibly injured myocardium. More studies are needed to elucidate the pathophysiologic mechanism of increased FDG uptake under fasting conditions in patients with infarcted myocardium.

Acknowledgements: The authors thank Dr. Brian D. Guth (Thomae, Essen, Germany) for reading the manuscript.

\section{References}

1) Brunken RC, Schwaiger M, Grover-McKay M, Phelps ME, Tillisch J, Schelbert HR. Positron emission tomography detects tissue metabolic activity in myocardial segments with persistent thallium perfusion defects. J Am Coll Cardiol 10: 557, 1987.

2) Tamaki N, Yonekura Y, Yamashita K, et al. Positron emission tomography using fluorine-18 deoxyglucose in evaluation of coronary artery bypass grafting. Am J Cardiol 64: 860, 1989.

3) Schwaiger M, Schelbert HR, Ellison D, et al. Sustained regional abnormalities in cardiac metabolism after transient ischemia in the chronic dog model. J Am Coll Cardiol 6: 336, 1985.

4) Schwaiger M, Brunken R, Grover-McKay M, et al. Regional myocardial metabolism in patients with acute myocardial infarction assessed by positron emission tomography. J Am Coll Cardiol 8: 800, 1986.

5) Chesebro JH, Kuatterud G, Roberts R, et al. Thrombolysis in myocardial infarction (TIMI) trial, phase 1: a comparison between intravenous tissue plasminogen activator and intravenous streptokinase. Circulation 76: 142, 1987.

6) Cohen M, Rentrop KP. Limitation of myocardial ischemia by collateral circulation during sudden controlled coronary occlusion in human subjects: a prospective study. Circulation 74: 469, 1986.

7) Brunken RC, Mody FV, Hawkins RA, Nienaber C, Phelps ME, Schelbert HR. Positron emission tomography defects metabolic viability in myocardium with persistent 24-hour single-photon emission computed tomography 201TL defects. Circulation 86: 1357, 1992.

8) Heger JJ, Weyman AE, Wann LS, Dillon JC, Feigenbaum H. Crosssectional echocardiography in acute myocardial infarction: detection and localization of regional left ventricular asynergy. Circulation 60: 531, 1979.

9) Melin JA, Wijns W, Keyeux A, et al. Assessment of thallium-201 redistribution versus glucose uptake as predictors of viability after coronary occlusion and reperfusion. Circulation 77: 927, 1988.

10) Sochor $\mathrm{H}$, Schwaiger M, Schelbert HR, et al. Relationship between TI201, Tc-99m (Sn) pyrophosphate and F-18 2-deoxyglucose uptake in ischemically injured dog myocardium. Am Heart J 114: 1066, 1987.

11) Camici P, Araujo LI, Spinks $T$, et al. Increased uptake of 18-Ffluorodeoxyglucose in postischemic myocardium of patients with exercise-induced angina. Circulation 74: 81, 1986.

12) Schwaiger M, Schelbert HR, Keen R, et al. Retention and clearance of C11 palmitic acid in ischemic and reperfused canine myocardium. J Am Coll Cardiol 6: 311, 1985.

13) Lopaschuk GD, Spafford MA, Davies NJ, Wall SR. Glucose and palmitate oxidation in isolated working rat hearts reperfused after a period of transient global ischemia. Circ Res 66: 546, 1990.

14) Liedtke AJ, Renstrom B, Nellis SH. Correlation between [5-3-H] glucose and [U-14-C] deoxyglucose as markers of glycolysis in reperfused myocardium. Circ Res 71: 689, 1992.

15) Wijns W, Melin JA, Leners N, et al. Accumulation of polymorphonuclear leukocytes in reperfused ischemic canine myocardium: relation with tissue viability assessed by fluorine-18-2-deoxy-glucose uptake. J Nucl Med 29: 1826, 1988.

16) Sease $D$, Garza D, Merhige ME, Gould KL. Does myocardial uptake of F-18-fluorodeoxyglucose by positron emission tomography reliably indicate myocardial viability in acute myocardial infarction? Circulation 80: (suppl II): II-378, 1989 (Abstract).

17) Sebree L, Bianco JA, Subramanian R, et al. Discordance between accumulation of C-14 deoxyglucose and TL-201 in reperfused myocardium. J Mol Cell Cardiol 23: 603, 1991.

18) Marwick TH, MacIntyre WJ, Lafont A, Nemec JJ, Salcedo EE. Metabolic responses of hibernating and infarcted myocardium to revascularization. A follow-up study of regional perfusion, function, and metabolism. Circulation 85: 1347, 1992.

19) The GISSI-3 APPI Study Group. Early and six-month outcome in patients with angina pectoris early after acute myocardial infarction (The GISSI3 APPI [Angina Precoce Post-Infarto] study). Am J Cardiol 78: 1191, 1996. 